\title{
A Topic Modeling Analysis of the Crisis Response Stage for COVID-19 Pandemic
}

\author{
Kyung-sook $\mathrm{CHA}^{\mathrm{a}}$ and Eun Man $\mathrm{KIM}^{\mathrm{b}, 1}$ \\ a Associate Professor, Department of Nursing, Sun Moon University \\ ${ }^{\mathrm{b}}$ Professor, Department of Nursing, Sun Moon University
}

\begin{abstract}
After the COVID-19 pandemic occurred in South Korea in 2020, medical institutions have dealt with the epidemiological crisis. The institutions' strategies in response to the crisis were classified into four stages depending on the change of epidemic circumstances. Efficiently responding to the pandemic, close cooperation between the government and medical institutions is essential.
\end{abstract}

Keywords. COVID-19, topic modeling, crisis response

\section{Introduction}

COVID-19 has been pandemic worldwide since it first broke out in China in December 2019 [1]. As medical institutions are public places where many patients are vulnerable to infection, fatal consequences can be easily caused when the emerging infectious disease is spread. Therefore, formulating thorough crisis-response strategies regarding the emerging infectious disease is necessary. The risk management of an epidemic must include both planned and improvised responses, which means the ability to respond to emergencies and compliance with manuals and guidelines is critical [2]. Identifying and organizing the countermeasure stages applied during the disaster period is the driving force for prompt response to similar crisis in the future. This study identified information provided to healthcare workers by infection control teams during the COVID-19 pandemic. The current research provides essential data needed for preparing measures to effectively cope with the crisis of emerging infectious disease that we may face in the future.

\section{Methods}

This study utilized text mining on big data to analyze the notices about COVID-19related in-hospital strategies. This study was conducted in the order of big data collection, preprocessing, and analysis, and the study implemented keyword analysis and topic modeling as an analytical method. As a result of searching for notices announced from January 1, 2020 to August 31, 2020 in the infection control teams of 12 university hospitals in five regions of South Korea, 1,653 cases and 50,567 sentences were identified. Stage was divided into four stages, taking into account the number of

\footnotetext{
${ }^{1}$ Corresponding Author, Eun Man Kim, Professor, Department of Nursing, Sun Moon University.
} 
confirmed COVID-19 cases, the spread pattern, and the government's 's stage of an infectious disease crisis. For the text network analysis, we aggregated the data to extract the top 110 120 words based on the total number of times the words appeared to create a word cloud, and ran a word cloud based on TF-IDF values created with the frequency of appearance and identifying only document-specific important words. Using the latent dirichlet allocation (LDA) technique, topic modeling analysis was performed by setting the number of topics to four, and setting MCMC > alpha 2.0, beta 0.001 , and no operation $=1000$.

\section{Results}

The analysis yielded four stages of topics as follow (Figure 1, Table 1).

Table 1. Classification by stage.

\begin{tabular}{|l|l|}
\hline Stage1 & $\begin{array}{l}\text { China, Novel Coronavirus Infections, Wuhan, Case definition, Pneumonia, Korea Centers for } \\
\text { Disease Control and Prevention (KCDC), Expansion, DUR, Entry, Community, report, } \\
\text { Information Response, Procedure, Announcement }\end{array}$ \\
\hline Stage2 & $\begin{array}{l}\text { Clinic, Screening Center, inhospital, PCR, Infectious disease declaration, Statistical Reporting } \\
\text { Consultation details, Occurrence status, Response Instructions, Inspection results, Process, } \\
\text { doctor } \\
\text { Diffusion, negative results }\end{array}$ \\
\hline Stage3 & $\begin{array}{l}\text { Confirmed cases, Movement line, Personal Protective Equipment, Novel Coronavirus } \\
\text { Infections } \\
\text { Notice, Examine, Situation Room, Level D, Dressing, movement line, doctor, Support } \\
\text { Specimen, Screening questionnaires }\end{array}$ \\
\hline Stage4 & $\begin{array}{l}\text { Novel Coronavirus Infections, doorway. Changes, Process, examine, Administration Process, } \\
\text { Guidelines } \\
\text { Response, coping, Visitors, Notice, Mobile, Diffusion, Control, Revision }\end{array}$ \\
\hline
\end{tabular}

Based on the keywords, the first-stage topic was named "Understanding the new infectious disease". This first stage is related to obtaining information about new infectious diseases, such as the definition of diseases, the methods of transmission, the areas of occurrence, and the legal reporting standards for new infectious diseases. The second-stage topic was named "Prepare a patient care and management system". The topic is about the process of establishing an overall system for patient selection, medical treatment, implementation of diagnostic tests, and confirmation of test results when suspected or confirmed patients visit medical institutions. The third-stage topic was named "Prevention of Diffusion and Employee Safety". The third stage is related to preventing the inflow of new coronavirus infections into medical institutions and strengthening employee safety to prevent the infection of faculty members who work on new suspected or confirmed coronavirus cases. With the keywords, the four-step topic was named "Improvement of the management system following the revision of the guidelines". As the epidemic period of COVID-19 is prolonged, the guidelines and standards provided by government agencies in the early stages have been revised and supplemented. 


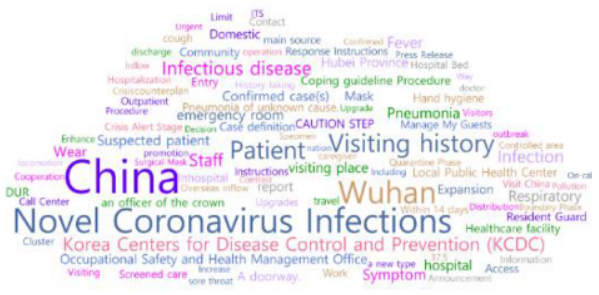

Stage 1

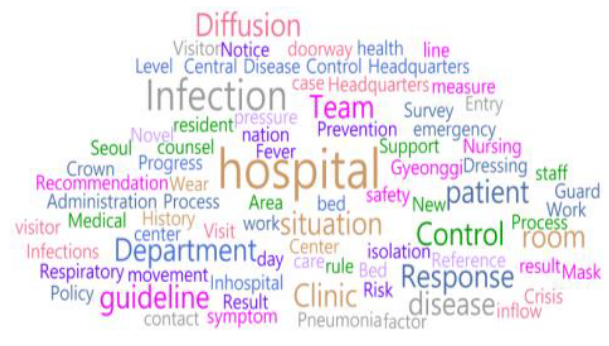

Stage3

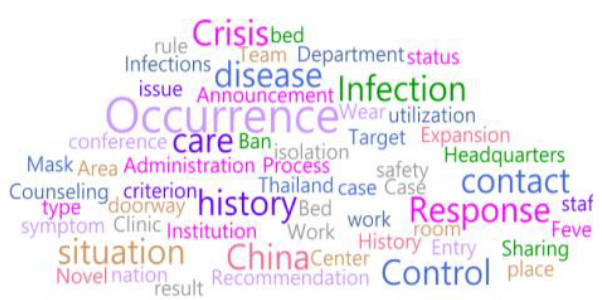

Stage2

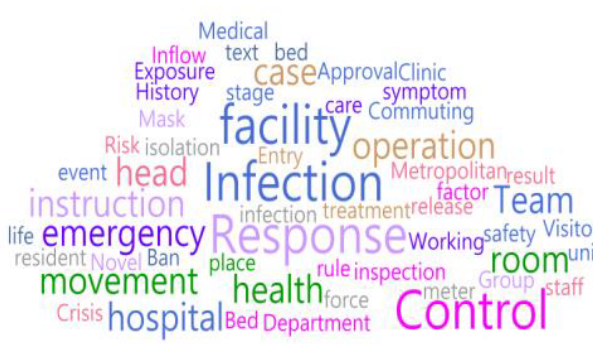

Stage4

Figure 1. The four stages.

\section{Conclusions}

As confirmed in the COVID-19 pandemic, an emerging infectious disease can have irreversible consequences if the initial response is immature. Where an emerging infectious disease occurs, a country shall provide accurate information on the disease and response guidelines, and medical institutions need to revise and complement them with the consideration of the institution's specific circumstances. Therefore, to respond efficiently in the event of an infectious disease crisis, the government and medical institutions must closely cooperate, and their systematic response is crucial.

\section{Acknowledgement}

This work was supported by the National Research Foundation of Korea (NRF) grant funded by the Korea government (MSIT) (No. 2020R1F1A1055437).

\section{References}

[1] World Health Organization. Origin of SARS-CoV-2, 26 March 2020. Available to. https://apps.who.int/iris/handle/10665/332197

[2] Farazmand A. Handbook of crisis and emergency management. 2001. Marcel Dekker, Inc., New York. 